\title{
QUANTITATIVE STRONG UNIQUE CONTINUATION FOR THE LAMÉ SYSTEM WITH LESS REGULAR COEFFICIENTS*
}

\author{
C.-L. LIN ${ }^{\dagger}$, G. NAKAMURA ${ }^{\ddagger}$, G. UHLMANN${ }^{\S}$ AND J.-N. WANG
}

\begin{abstract}
In this paper we prove a quantitative form of the strong unique continuation property for the Lamé system when the Lamé coefficients $\mu$ is Lipschitz and $\lambda$ is essentially bounded in dimension $n \geq 2$. This result is an improvement of our earlier result [5] in which both $\mu$ and $\lambda$ were assumed to be Lipschitz.
\end{abstract}

Key words. Lame system, strong unique continuation property, Carleman estimates.

AMS subject classifications. Primary 35Q72; Secondary 35J55.

1. Introduction. Assume that $\Omega$ is a connected open set containing 0 in $\mathbb{R}^{n}$ for $n \geq 2$. Let $\mu(x) \in C^{0,1}(\Omega)$ and $\lambda(x), \rho(x) \in L^{\infty}(\Omega)$ satisfy

$$
\left\{\begin{array}{l}
\mu(x) \geq \delta_{0}, \quad \lambda(x)+2 \mu(x) \geq \delta_{0} \quad \forall \text { a.e. } x \in \Omega, \\
\|\mu\|_{C^{0,1}(\Omega)}+\|\lambda\|_{L^{\infty}(\Omega)} \leq M_{0}, \quad\|\rho\|_{L^{\infty}(\Omega)} \leq M_{0}
\end{array}\right.
$$

with positive constants $\delta_{0}, M_{0}$, where we define

$$
\|f\|_{C^{0,1}(\Omega)}=\|f\|_{L^{\infty}(\Omega)}+\|\nabla f\|_{L^{\infty}(\Omega)} .
$$

The isotropic elasticity system, which represents the displacement equation of equilibrium, is given by

$$
\operatorname{div}\left(\mu\left(\nabla u+(\nabla u)^{t}\right)\right)+\nabla(\lambda \operatorname{div} u)+\rho u=0 \quad \text { in } \Omega,
$$

where $u=\left(u_{1}, u_{2}, \cdots, u_{n}\right)^{t}$ is the displacement vector and $(\nabla u)_{j k}=\partial_{k} u_{j}$ for $j, k=$ $1,2, \cdots, n$.

We are interested in the strong unique continuation property (SUCP) of (1.2). We say that the solution $u$ of (1.2) satisfies the SUCP if $u$ that vanishes of infinite order at $x_{0} \in \Omega$ vanishes identically in $\Omega$. In other words, if $u$ of (1.2) satisfies that

$$
\int_{\left|x-x_{0}\right|<R}|u|^{2} d x=O\left(R^{N}\right) \quad \forall \quad N \in \mathbb{N}
$$

for all sufficiently small $R$, then $u \equiv 0$ in $\Omega$. On the other hand, we say that $u$ of (1.2) satisfies the unique continuation property (UCP) if $u=0$ in a nonempty open subset of $\Omega$ implies that $u \equiv 0$ in $\Omega$. It is obvious that SUCP implies UCP. In this paper, we would like to show that any nontrivial solution of (1.2) can only vanish of finite order at any point of $\Omega$. We also give an estimate of the vanishing order for $u$, which can be seen as a quantitative description of the SUCP for (1.2). Here we list some of the known results on the SUCP for (1.2):

\footnotetext{
*Received December 31, 2010; accepted for publication March 17, 2011.

$\dagger$ Department of Mathematics, NCTS, National Cheng Kung University, Tainan 701, Taiwan (cllin2@mail.ncku.edu.tw).

${ }^{\ddagger}$ Department of Mathematics, Hokkaido University, Sapporo 060-0810, Japan (gnaka@math.sci. hokudai.ac.jp).

$\S$ Department of Mathematics, University of Washington, Seattle, WA 98195 and University of California, Irvine, CA 92697-3875, USA (gunther@math.washington.edu).

『Department of Mathematics, Taida Institute of Mathematical Sciences, NCTS (Taipei), National Taiwan University, Taipei 106, Taiwan (jnwang@math.ntu.edu.tw).
} 
- $\lambda, \mu \in C^{1,1}, n \geq 2$ (quantitative): Alessandrini and Morassi [1].

- $\lambda, \mu \in C^{0,1}, n=2$ (qualitative): Lin and Wang [4].

- $\lambda \in L^{\infty}, \mu \in C^{0,1}, n=2$ (qualitative): Escauriaza [2].

- $\lambda, \mu \in C^{0,1}, n \geq 2$ (quantitative): Lin, Nakamura, and Wang [5].

In this paper, we relax the regularity assumption on $\lambda$ in [5] to $\lambda \in L^{\infty}(\Omega)$. In view of counterexamples by Plis [7] or Miller [3], this regularity assumption seems to be optimal. This improvement was inspired by our recent work on the Stokes system [6]. We now state the main results of the paper. Assume that there exists $0<R_{0} \leq 1$ such that $B_{R_{0}} \subset \Omega$. Hereafter $B_{r}$ denotes an open ball of radius $r>0$ centered at the origin.

THEOREM 1.1 (Optimal three-ball inequalities). There exists a positive number $\tilde{R}<1$, depending only on $n, M_{0}, \delta_{0}$, such that if $0<R_{1}<R_{2}<R_{3} \leq R_{0}$ and $R_{1} / R_{3}<R_{2} / R_{3}<\tilde{R}$, then

$$
\int_{|x|<R_{2}}|u|^{2} d x \leq C\left(\int_{|x|<R_{1}}|u|^{2} d x\right)^{\tau}\left(\int_{|x|<R_{3}}|u|^{2} d x\right)^{1-\tau}
$$

for $u \in H_{\text {loc }}^{1}\left(B_{R_{0}}\right)$ satisfying (1.2) in $B_{R_{0}}$, where the constant $C$ depends on $R_{2} / R_{3}$, $n, M_{0}, \delta_{0}$, and $0<\tau<1$ depends on $R_{1} / R_{3}, R_{2} / R_{3}, n, M_{0}, \delta_{0}$. Moreover, for fixed $R_{2}$ and $R_{3}$, the exponent $\tau$ behaves like $1 /\left(-\log R_{1}\right)$ when $R_{1}$ is sufficiently small.

As in [5], we can derive the lower bound of $\int_{|x|<R}|u|^{2} d x$ from Theorem 1.1. We refer the reader to [5] for more details.

THEOREM 1.2. Let $u \in H_{l o c}^{1}(\Omega)$ be a nontrivial solution of $(1.2)$, then there exist positive constants $K$ and $m$, depending on $n, M_{0}, \delta_{0}$ and $u$, such that

$$
\int_{|x|<R}|u|^{2} d x \geq K R^{m}
$$

for all $R$ sufficiently small.

REMARK 1.3. Based on Theorem 1.1, the constants $K$ and $m$ in (1.4) are explicitly given by

$$
K=\int_{|x|<R_{3}}|u|^{2} d x
$$

and

$$
m=\tilde{C}+\log \left(\frac{\int_{|x|<R_{3}}|u|^{2} d x}{\int_{|x|<R_{2}}|u|^{2} d x}\right),
$$

where $\tilde{C}$ is a positive constant depending on $n, M_{0}, \delta_{0}$ and $R_{2} / R_{3}$.

From (1.4), we have that $u$ can only vanish of finite order at the origin. In fact, we can prove the following stronger version of SUCP.

Corollary 1.4. Let $u \in H_{\text {loc }}^{1}(\Omega)$ be a solution of (1.2) and for some $N>m$, where $m$ is the constant given in Theorem 1.2,

$$
\int_{|x|<R}|u|^{2} d x=O\left(R^{N}\right), \quad \text { for all sufficiently small } R,
$$

then $u \equiv 0$ in $\Omega$. 
2. Reduced system and estimates. Here we want to find a reduced system from (1.2). This is a crucial step in our approach. Let us write (1.2) into a nondivergence form:

$$
\mu \Delta u+\nabla((\lambda+\mu) \operatorname{div} u)+\left(\nabla u+(\nabla u)^{t}\right) \nabla \mu-\operatorname{div} u \nabla \mu+\rho u=0 .
$$

Dividing (2.1) by $\mu$ yields

$$
\begin{aligned}
& \Delta u+\frac{1}{\mu} \nabla((\lambda+\mu) \operatorname{div} u)+\left(\nabla u+(\nabla u)^{t}\right) \frac{\nabla \mu}{\mu}-\operatorname{div} u \frac{\nabla \mu}{\mu}+\frac{\rho}{\mu} u \\
= & \Delta u+\nabla\left(\frac{\lambda+\mu}{\mu} \operatorname{div} u\right)+\left(\nabla u+(\nabla u)^{t}\right) \frac{\nabla \mu}{\mu}-\operatorname{div} u\left(\frac{\nabla \mu}{\mu}+(\lambda+\mu) \nabla\left(\frac{1}{\mu}\right)\right) \\
& +\frac{\rho}{\mu} u \\
= & \Delta u+\nabla(a(x) v)+G \\
(2.2)= & 0,
\end{aligned}
$$

where

$$
a(x)=\frac{\lambda+\mu}{\lambda+2 \mu} \in L^{\infty}(\Omega), \quad v=\frac{\lambda+2 \mu}{\mu} \operatorname{div} u
$$

and

$$
G=\left(\nabla u+(\nabla u)^{t}\right) \frac{\nabla \mu}{\mu}-\operatorname{div} u\left(\frac{\nabla \mu}{\mu}+(\lambda+\mu) \nabla\left(\frac{1}{\mu}\right)\right)+\frac{\rho}{\mu} u .
$$

Taking the divergence on (2.2) gives

$$
\Delta v+\operatorname{div} G=0 .
$$

Our reduced system now consists of (2.2) and (2.3). It follows easily from (2.3) that if $u \in H_{l o c}^{1}(\Omega)$, then $v \in H_{l o c}^{1}(\Omega)$.

To prove the main results, we rely on suitable Carleman estimates. Denote $\varphi_{\beta}=$ $\varphi_{\beta}(x)=\exp (-\beta \tilde{\psi}(x))$, where $\beta>0$ and $\tilde{\psi}(x)=\log |x|+\log \left((\log |x|)^{2}\right)$. Note that $\varphi_{\beta}$ is less singular than $|x|^{-\beta}$. We use the notation $X \lesssim Y$ or $X \gtrsim Y$ to mean that $X \leq C Y$ or $X \geq C Y$ with some constant $C$ depending only on $n$.

LEMma 2.1. [5, Lemma 2.4] There exist a sufficiently small number $r_{1}>0$ depending on $n$ and a sufficiently large number $\beta_{1}>3$ depending on $n$ such that for all $w \in U_{r_{1}}$ and $f=\left(f_{1}, \cdots, f_{n}\right) \in\left(U_{r_{1}}\right)^{n}, \beta \geq \beta_{1}$, we have that

$$
\begin{aligned}
& \int \varphi_{\beta}^{2}(\log |x|)^{2}\left(\beta|x|^{4-n}|\nabla w|^{2}+\beta^{3}|x|^{2-n}|w|^{2}\right) d x \\
\lesssim & \int \varphi_{\beta}^{2}(\log |x|)^{4}|x|^{2-n}\left[\left(|x|^{2} \Delta w+|x| \operatorname{div} f\right)^{2}+\beta^{2}\|f\|^{2}\right] d x,
\end{aligned}
$$

where $U_{r_{1}}=\left\{w \in C_{0}^{\infty}\left(\mathbb{R}^{n} \backslash\{0\}\right): \operatorname{supp}(w) \subset B_{r_{0}}\right\}$.

Next, replacing $\beta$ by $\beta+1$ in (2.4) and note that

$$
\varphi_{\beta+1}^{2}=\varphi_{\beta}^{2}|x|^{-2}(\log |x|)^{4} .
$$

Then, we can get another Carleman estimate. 
LEMMA 2.2. There exist a sufficiently small number $r_{1}>0$ depending on $n$ and a sufficiently large number $\beta_{1}>2$ depending on $n$ such that for all $w \in U_{r_{1}}$ and $f=\left(f_{1}, \cdots, f_{n}\right) \in\left(U_{r_{1}}\right)^{n}, \beta \geq \beta_{1}$, we have that

$$
\begin{aligned}
& \int \varphi_{\beta}^{2}(\log |x|)^{-2}\left(\beta|x|^{2-n}|\nabla w|^{2}+\beta^{3}|x|^{-n}|w|^{2}\right) d x \\
\lesssim & \int \varphi_{\beta}^{2}|x|^{-n}\left[\left(|x|^{2} \Delta w+|x| \operatorname{div} f\right)^{2}+\beta^{2}\|f\|^{2}\right] d x .
\end{aligned}
$$

In addition to Carleman estimates, we also need the following Caccioppoli's type inequality.

LEMMA 2.3. Let $u \in\left(H_{\text {loc }}^{1}(\Omega)\right)^{n}$ be a solution of (1.1). Then for any $0<a_{3}<$ $a_{1}<a_{2}<a_{4}$ such that $B_{a_{4} r} \subset \Omega$ and $\left|a_{4} r\right|<1$, we have

$$
\int_{a_{1} r<|x|<a_{2} r}|x|^{4}|\nabla v|^{2}+|x|^{2}|v|^{2}+|x|^{2}|\nabla u|^{2} d x \leq C_{0} \int_{a_{3} r<|x|<a_{4} r}|u|^{2} d x
$$

where the constant $C_{0}$ is independent of $r$ and $u$. Here $v$ is defined in (2.2).

The proof of Lemma 2.3 will be given in the next section. Here we would like to outline how to proceed the proofs of main theorems. The detailed arguments can be found in [5] or [6]. Firstly, applying (2.5) to $w=u, f=|x| a(x) v$ and using (2.2), we have that

$$
\begin{aligned}
& \int \varphi_{\beta}^{2}(\log |x|)^{-2}\left(\beta|x|^{2-n}|\nabla u|^{2}+\beta^{3}|x|^{-n}|u|^{2}\right) d x \\
\lesssim & \int \varphi_{\beta}^{2}|x|^{-n}\left[\left(|x|^{2} \Delta u+|x| \operatorname{div}(|x| a(x) v)\right)^{2}+\beta^{2}\||x| a(x) v\|^{2}\right] d x .
\end{aligned}
$$

Next, applying (2.4) to $w=v, f=|x| G$ and using (2.3), we get that

$$
\begin{aligned}
& \int \varphi_{\beta}^{2}(\log |x|)^{2}\left(\beta|x|^{4-n}|\nabla v|^{2}+\beta^{3}|x|^{2-n}|v|^{2}\right) d x \\
\lesssim & \int \varphi_{\beta}^{2}(\log |x|)^{4}|x|^{2-n}\left[\left(|x|^{2} \Delta v+|x| \operatorname{div}(|x| G)\right)^{2}+\beta^{2}\||x| G\|^{2}\right] d x .
\end{aligned}
$$

Finally, adding $\beta \times(2.7)$ and (2.8) together and using (2.6), we can prove Theorem 1.1 and 1.2 as in [5] and [6].

3. Proof of Lemma 2.3. Define $b_{1}=\left(a_{1}+a_{3}\right) / 2$ and $b_{2}=\left(a_{2}+a_{4}\right) / 2$. Let $X=B_{a_{4} r} \backslash \bar{B}_{a_{3} r}, Y=B_{b_{2} r} \backslash \bar{B}_{b_{1} r}$ and $Z=B_{a_{2} r} \backslash \bar{B}_{a_{1} r}$. Let $\xi(x) \in C_{0}^{\infty}\left(\mathbb{R}^{n}\right)$ satisfy $0 \leq \xi(x) \leq 1$ and

$$
\xi(x)= \begin{cases}0, & |x| \leq a_{3} r \\ 1, & b_{1} r<|x|<b_{2} r \\ 0, & |x| \geq a_{4} r\end{cases}
$$


From (1.2), we have that

$$
\begin{aligned}
0= & -\int\left[\operatorname{div}\left(\mu\left(\nabla u+(\nabla u)^{t}\right)\right)+\nabla(\lambda \operatorname{div} u)+\rho u\right] \cdot\left(\xi^{2} \bar{u}\right) d x \\
= & \int \sum_{i j k l=1}^{n}\left[\lambda \delta_{i j} \delta_{k l}+\mu\left(\delta_{i l} \delta_{j k}+\delta_{i k} \delta_{j l}\right)\right] \partial_{x_{l}} u_{k} \partial_{x_{j}}\left(\xi^{2} \bar{u}_{i}\right) d x-\int \rho \xi^{2}|u|^{2} d x \\
= & \int \xi^{2} \sum_{i j k l=1}^{n}\left[\lambda \delta_{i j} \delta_{k l}+\mu\left(\delta_{i l} \delta_{j k}+\delta_{i k} \delta_{j l}\right)\right] \partial_{x_{l}} u_{k} \partial_{x_{j}} \bar{u}_{i} d x \\
& +\int \sum_{i j k l=1}^{n} \partial_{x_{j}}\left(\xi^{2}\right)\left[\lambda \delta_{i j} \delta_{k l}+\mu\left(\delta_{i l} \delta_{j k}+\delta_{i k} \delta_{j l}\right)\right] \partial_{x_{l}} u_{k} \bar{u}_{i} d x-\int \rho \xi^{2}|u|^{2} d x \\
(3.2)= & I_{1}+I_{2},
\end{aligned}
$$

where

$$
I_{1}=\int \xi^{2}\left[\sum_{i j=1}^{n} \lambda \partial_{x_{j}} u_{j} \partial_{x_{i}} \bar{u}_{i}+\sum_{i j=1}^{n} \mu\left(\partial_{x_{i}} u_{j} \partial_{x_{j}} \bar{u}_{i}+\partial_{x_{j}} u_{i} \partial_{x_{j}} \bar{u}_{i}\right)\right] d x
$$

and

$$
I_{2}=\int \sum_{i j k l=1}^{n} \partial_{x_{j}}\left(\xi^{2}\right)\left[\lambda \delta_{i j} \delta_{k l}+\mu\left(\delta_{i l} \delta_{j k}+\delta_{i k} \delta_{j l}\right)\right] \partial_{x_{l}} u_{k} \bar{u}_{i} d x-\int \rho \xi^{2}|u|^{2} d x
$$

Observe that

$$
\begin{aligned}
& \int \xi^{2}\left(2 \mu-\frac{\delta_{0}}{2}\right) \partial_{x_{i}} u_{j} \partial_{x_{j}} \bar{u}_{i} d x \\
= & -\int \partial_{x_{j}}\left[\xi^{2}\left(2 \mu-\frac{\delta_{0}}{2}\right)\right] \partial_{x_{i}} u_{j} \bar{u}_{i} d x-\int \xi^{2}\left(2 \mu-\frac{\delta_{0}}{2}\right) \partial_{x_{i} x_{j}}^{2} u_{j} \bar{u}_{i} d x \\
= & -\int \partial_{x_{j}}\left[\xi^{2}\left(2 \mu-\frac{\delta_{0}}{2}\right)\right] \partial_{x_{i}} u_{j} \bar{u}_{i} d x+\int \partial_{x_{i}}\left[\xi^{2}\left(2 \mu-\frac{\delta_{0}}{2}\right)\right] \partial_{x_{j}} u_{j} \bar{u}_{i} d x \\
& +\int \xi^{2}\left(2 \mu-\frac{\delta_{0}}{2}\right) \partial_{x_{j}} u_{j} \partial_{x_{i}} \bar{u}_{i} d x
\end{aligned}
$$


It follows from (3.3) that

$$
\begin{aligned}
I_{1}= & \int \xi^{2}\left[\sum_{i j=1}^{n} \lambda \partial_{x_{j}} u_{j} \partial_{x_{i}} \bar{u}_{i}+\sum_{i j=1}^{n}\left(2 \mu-\frac{\delta_{0}}{2}\right)\left(\partial_{x_{i}} u_{j} \partial_{x_{j}} \bar{u}_{i}\right)\right] d x \\
& +\int \sum_{i j=1}^{n} \xi^{2}\left(\mu-\frac{\delta_{0}}{2}\right)\left(\partial_{x_{j}} u_{i} \partial_{x_{j}} \bar{u}_{i}-\partial_{x_{i}} u_{j} \partial_{x_{j}} \bar{u}_{i}\right) d x \\
& +\frac{\delta_{0}}{2} \int \sum_{i j=1}^{n} \xi^{2} \partial_{x_{j}} u_{i} \partial_{x_{j}} \bar{u}_{i} d x \\
= & \int\left(2 \mu+\lambda-\frac{\delta_{0}}{2}\right) \xi^{2} \sum_{i j=1}^{n}\left(\partial_{x_{j}} u_{j} \partial_{x_{i}} \bar{u}_{i}\right) d x \\
& +\int \sum_{i j=1}^{n} \xi^{2}\left(\mu-\frac{\delta_{0}}{2}\right)\left(\partial_{x_{j}} u_{i} \partial_{x_{j}} \bar{u}_{i}-\partial_{x_{i}} u_{j} \partial_{x_{j}} \bar{u}_{i}\right) d x \\
& +\frac{\delta_{0}}{2} \int \sum_{i j=1}^{n} \xi^{2} \partial_{x_{j}} u_{i} \partial_{x_{j}} \bar{u}_{i} d x+I_{3},
\end{aligned}
$$

where

$$
I_{3}=\sum_{i j=1}^{n} \int \partial_{x_{i}}\left[\xi^{2}\left(2 \mu-\frac{\delta_{0}}{2}\right)\right] \partial_{x_{j}} u_{j} \bar{u}_{i}-\partial_{x_{j}}\left[\xi^{2}\left(2 \mu-\frac{\delta_{0}}{2}\right)\right] \partial_{x_{i}} u_{j} \bar{u}_{i} d x
$$

Since

$$
\begin{aligned}
& \int \sum_{i j=1}^{n} \xi^{2}\left(\mu-\frac{\delta_{0}}{2}\right)\left(\partial_{x_{j}} u_{i} \partial_{x_{j}} \bar{u}_{i}-\partial_{x_{i}} u_{j} \partial_{x_{j}} \bar{u}_{i}\right) d x \\
= & \frac{1}{2} \int \sum_{i j=1}^{n} \xi^{2}\left(\mu-\frac{\delta_{0}}{2}\right)\left|\partial_{x_{j}} u_{i}-\partial_{x_{i}} u_{j}\right|^{2} d x
\end{aligned}
$$

we obtain that

$$
I_{1} \geq \frac{\delta_{0}}{2} \int|\xi \nabla u|^{2} d x+I_{3}
$$

Combining (3.2) and (3.5), we have that

$$
\int_{Y}|\nabla u|^{2} d x \leq \int_{X}|\xi \nabla u|^{2} d x \leq C_{1} \int_{X}|x|^{-2}|u|^{2} d x
$$

which implies

$$
\int_{Y}|x|^{2}|\nabla u|^{2} d x \leq C_{2} \int_{X}|u|^{2} d x
$$

Here and below all constants $C_{1}, C_{2}, \cdots$ depend on $\delta_{0}, M_{0}$.

To estimate $\nabla v$, we define $\chi(x) \in C_{0}^{\infty}\left(\mathbb{R}^{n}\right)$ satisfy $0 \leq \chi(x) \leq 1$ and

$$
\chi(x)= \begin{cases}0, & |x| \leq b_{1} r \\ 1, & a_{1} r<|x|<a_{2} r \\ 0, & |x| \geq b_{2} r\end{cases}
$$


By (2.3), we derive that

$$
\begin{aligned}
& \int|\chi(x) \nabla v|^{2} d x \\
= & \int \nabla v \cdot \nabla\left(\chi^{2} \bar{v}\right) d x-2 \int \chi \nabla v \cdot \bar{v} \nabla \chi d x \\
\leq & \left|\int(\operatorname{div} G) \chi^{2} \bar{v} d x\right|+2 \int|\chi \nabla v \cdot \bar{v} \nabla \chi| d x \\
\leq & \left|\int(\operatorname{div} G) \chi^{2} \bar{v} d x\right|+\frac{1}{4} \int|\chi \nabla v|^{2} d x+C_{3} \int_{Y}|x|^{-2}|v|^{2} d x \\
\leq & C_{4} \int_{Y}|\nabla u|^{2} d x+C_{4} \int_{Y}|u|^{2} d x+\frac{1}{2} \int|\chi \nabla v|^{2} d x+C_{4} \int_{Y}|x|^{-2}|v|^{2} d x \\
\leq & C_{5} \int_{Y}|x|^{-2}|\nabla u|^{2} d x+C_{4} \int_{Y}|u|^{2} d x+\frac{1}{2} \int|\chi \nabla v|^{2} d x .
\end{aligned}
$$

Therefore, we get from (3.7) that

$$
\int_{Z}|\nabla v|^{2} d x \leq 2 C_{5} \int_{Y}|x|^{-2}|\nabla u|^{2} d x+2 C_{4} \int_{Y}|u|^{2} d x
$$

and hence

$$
\int_{Z}|x|^{4}|\nabla v|^{2} d x \leq C_{6} \int_{Y}|x|^{2}|\nabla u|^{2} d x+C_{6} \int_{Y}|x|^{4}|u|^{2} d x .
$$

Putting together $K \times(3.6)$ and (3.8), we have that

$$
\begin{aligned}
& K \int_{Y}|x|^{2}|\nabla u|^{2} d x+\int_{Z}|x|^{4}|\nabla v|^{2} d x \\
\leq & K C_{2} \int_{X}|u|^{2} d x+C_{6} \int_{Y}|x|^{2}|\nabla u|^{2} d x+C_{6} \int_{Y}|x|^{4}|u|^{2} d x .
\end{aligned}
$$

Choosing $K=2 C_{6}$ in $(3.9)$ yields

$$
\begin{aligned}
& \int_{Z}|x|^{2}|v|^{2} d x+\int_{Z}|x|^{2}|\nabla u|^{2} d x+\int_{Z}|x|^{4}|\nabla v|^{2} d x \\
\leq & C_{7} \int_{Y}|x|^{2}|\nabla u|^{2} d x+C_{7} \int_{Z}|x|^{4}|\nabla v|^{2} d x \\
\leq & C_{8} \int_{X}|u|^{2} d x,
\end{aligned}
$$

The proof is now complete.

Acknowledgements. Lin was supported in part by the National Science Council of Taiwan. Nakamura was partially supported by Grant-in-Aid for Scientific Research (B)(No.19340028) of Japan Society for Promotion of Science. Uhlmann was supported in part by NSF and Senior Clay Award and Chancellor Professorship at UC Berkeley. Wang was partially supported by the National Science Council of Taiwan. 


\section{REFERENCES}

[1] G. Alessandrini And A. Morassi, Strong unique continuation for the Lamé system of elasticity, Comm. in PDE., 26 (2001), pp. 1787-1810.

[2] L. EscauriazA, Unique continuation for the system of elasticity in the plane, Proc. Amer. Math. Soc., 134 (2006), pp. 2015-2018.

[3] K. Miller, Nonunique continuation for uniformly parabolic and elliptic equations in selfadjoint divergence form with Hölder continuous coefficients, Bull. AMS, 79 (1973), pp. 350-354.

[4] C. L. Lin ANd J. N. Wang, Strong unique continuation for the Lamé system with Lipschitz coefficients, Math. Ann., 331 (2005), pp. 611-629.

[5] C. L. Lin, G. Nakamura And J. N. Wang, Optimal three-ball inequalities and quantitative uniqueness for the Lamé system with Lipschitz coefficients, Duke Math Journal, 155:1 (2010), pp. 189-204.

[6] C. L. Lin, G. Uhlmann, And J. N. WANG, Optimal three-ball inequalities and quantitative uniqueness for the Stokes system, DCDS-A (Special issue dedicated to Professor Louis Nirenberg on the occasion of his 85th birthday), 28 (2010), pp. 1273-1290.

[7] A. PLIS, On non-uniqueness in Cauchy problem for an elliptic second order differential equation, Bull. Acad. Polon. Sci. Ser. Sci. Math. Astronom. Phys., 11 (1963), pp. 95-100. 